\title{
RGV dynamic scheduling optimization model based on greedy algorithm
}

\author{
Wang Haoze ${ }^{1}$, Dong Chen ${ }^{2}$, Xu Yuan ${ }^{3}$ \\ ${ }^{1}$ School of computer and information technology, Beijing Jiaotong University, Beijing,264401 \\ ${ }^{2}$ School of electronic information engineering, Beijing Jiaotong University, Beijing,264401 \\ ${ }^{3}$ School of economics and management, Beijing Jiaotong University, Beijing,264401
}

Keywords: Greedy algorithm; RGV dynamic scheduling; global optimal

Summary: For one intelligent processing system which inclusionRGV, how to effectively use various resources to rationally and dynamically perform dynamic scheduling to improve production efficiency is the key. This paper studies only the material processing operations of a single process. According to the processing process of a given material, we need to focus on analyzing its dynamic scheduling strategy. In a material processing system with an established 8-hour working time, maximizing the amount of material processing is the primary goal. However, the increased amount of material processing is obtained by continuously completing the accumulation of work tasks. Therefore, the core is to convert the material processing quantity maximization model in the system into a task selection planning model based on time loss minimization, and seek each with a greedy algorithm. A task selects the optimal solution locally, and approximates each local optimal combination as a global optimal.

\section{Introduction}

RGV is an unmanned, intelligent track-type automatic guided vehicle that can run freely on a fixed track. It can automatically control the moving direction and distance according to the instructions to complete loading and unloading and cleaning materials. For one intelligent processing system which inclusionRGV, how to effectively use various resources to reasonably improve dynamic scheduling to improve production efficiency is the key.

\section{Model establishment}

For industrial processing systems, the amount of material processed during working hours has always been the primary goal.NIndicates the total quantity of material processing during working hours. Since each material that has been processed needs to be cleaned, replace the material processing quantity with the number of material cleaning times during working hours, and establish the following integer programming model.

$$
\max Z=N
$$




$$
\left\{\begin{array}{c}
N=\sum_{i=1}^{m} x_{i} \\
x_{i}=\left\{\begin{array}{c}
1 \text { The } i-\text { th task contains material cleaning } \\
0 \text { The } i-\text { th task does not contain material cleaning }
\end{array}\right.
\end{array}\right.
$$

Among them $\mathrm{m}$ indicates that during 8 hours of working time The total RGV number of tasks performed.

The increase in the number of material processing is accumulated by the completion of one material processing task. If the material processing quantity is as high as possible, Then the processing time of each CNC should be as long as possible, that is, the loss of time should be as small as possible., so for each task $\mathrm{P}_{\mathrm{i}}$ To determine, the goal is to establish a linear programming model with the least time loss as follows:

$$
\begin{gathered}
\min Z=W\left(P_{i}\right) \\
\left\{\begin{array}{c}
W\left(P_{i}\right)=n t_{\text {run }}+(n-1) t_{P_{i}} \\
t_{\text {run }}=t_{u j} \\
P_{i} \in W \\
t_{P_{i}} \in\left\{t_{i}, t_{i}+t_{w}\right\} \\
n \in\{1,2,3,4,5,6,7,8\} \\
t_{\text {run }}, t_{P_{i}}>0
\end{array}\right.
\end{gathered}
$$

\section{Greedy algorithm}

The greedy algorithm is an improved hierarchical processing method. The greedy algorithm is characterized by a greedy strategy, step by step, and each step is guaranteed to obtain a local optimal solution. In the above model establishment, it is a greedy idea to indirectly realize the maximum amount of processed materials by minimizing the time loss of each task selection. The greedy strategy is the strategy that minimizes the time loss generated after each task is selected. Based on such a strategy, the local optimal solution is obtained, and then the global optimal is solved.

The steps to give a dynamic scheduling algorithm based on greedy algorithm are as follows:

Step1: Start work, total material processingN $=0$ If the CNC is in idle state, the demand signal for feeding is sent to the RGV; if the processing operation is completed, the demand signal is sent out, and the task set at this time is, at this time RGV task set is $M=\left\{M_{1}, M_{2}, \cdots, M_{n}\right\}$

Step2: Calculate the loss function value for each task separatelyW $\left(\mathrm{M}_{\mathrm{i}}\right),\left(\mathrm{M}_{\mathrm{i}}\right) \in \mathrm{M}$

Step3: Select the following conditions $\mathrm{M}_{\mathrm{i}}$

$$
\mathrm{W}\left(\mathrm{M}_{\mathrm{i}}\right)=\min \left\{\mathrm{W}\left(\mathrm{M}_{\mathrm{j}}\right) \mid \mathrm{M}_{\mathrm{j}} \in \mathrm{M}\right\}
$$

Step4: Will task $M_{i}$ determine the task to be executed for the next step, judge the task $M_{i}$, Whether it contains the cleaning of the material, if it contains the total processing of the materialN,$++ \mathrm{M}_{\mathrm{i}} \mathrm{After}$ the execution is completed, calculate the working time and generate a new one.RGVTask collectionM, repeat Step2, Step3, Step4.

Step5: If the working time exceeds8Hours, stop task selection, calculate the number of processed materials, and save specific scheduling results.

\section{Model practicality test}

For the first eight CNC-numbering operations, 1,2,3,4,5,6,7,8 can be obtained quickly by greedy algorithm. RGV is between No. 7 and No. 8 A after the completion of the 8th material feeding. By 
simple calculation, RGV will not receive any demand signal from CNC. At this time, it is necessary to wait in situ until No. 1 CNC processing material is completed to send out the demand signal.

When RGV returns to the position between No.7 and No.8,the material processed in No.1 C NC is not yet finished and needs to wait in situ,so the subsequent material processing can be carried out in reciprocating o rder of 1,2,3,4,5,6,7,8 for loading and unloading and clinker cleaning.In the cyclic state,the dynamic scheduling results can be guaranteed to be optimal,and there is no need to continue to optimize.

For the determination of each task, the time loss is no longer the minimum as the task selection criterion, based on the principle of first come first served (FIFOThe task selection solution is solved, and the total number of materials processed based on the three groups of data in the 8-hour working time is obtained.

Table 1 Three sets of data FIFO principle material processing total table

\begin{tabular}{cccc}
$\begin{array}{c}\text { Task selection } \\
\text { principle }\end{array}$ & $\begin{array}{c}\text { Number of material } \\
\text { processing based on } \\
\text { the first set of data }\end{array}$ & $\begin{array}{c}\text { Number of material } \\
\text { processing based } \\
\text { on the second set of } \\
\text { data }\end{array}$ & $\begin{array}{c}\text { Number of } \\
\text { material } \\
\text { processing based } \\
\text { on the third set of } \\
\text { data }\end{array}$ \\
\hline FIFO & 356 & 337 & 366 \\
\hline
\end{tabular}

\section{Conclusion}

The greedy algorithm-based model involved in this paper is easier to implement and requires less computational resources, so it is often used to solve problems. At the same time, the model can also be used in the problem of finding the minimum distance, such as railway track construction, national road construction, overpass construction and network construction between two internal networks.

\section{References}

[1] Qiao Fei, Wu Qidi. Real-time Scheduling and Fault Scheduling of RGV in SJ-FMS[J]. Combined Machine Tool \& Automatic Processing Technology, 1995(3): 39-43.

[2] Li Cunchang, Li Haolin. Overall Design and Dynamic Real-time Scheduling of Automatic Guide Vehicle (AGV)[J]. Combined Machine Tool \& Automatic Processing Technology, 1996(4): 32-36. 